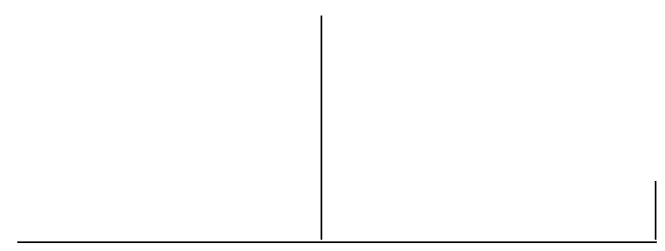

Rev. Latinoam. Psicopat. Fund., IX, 3, 387-409

\title{
Le trauma à l'épreuve de l'espace transféro-transitionnel Freud, Lacan, Winnicott et quelques autres...
}

\author{
Jacques Cabassut \\ Mohammed Ham
}

L' approche freudo-lacanienne, parallèlement à la mauvaise rencontre du réel, définit le traumatisme en tant qu'effet de langage potentiellement structurant pour le sujet. La pensée winnicottienne, paradoxale par excellence, offre quant à elle au jeu ou au lien précoce de l'infans à la "bonne mère", une place centrale au détriment de la pulsion, de la sexualité infantile, de l'aspect langagier, ou du rôle du père concernant le trauma.

En formulant un espace transféro-transitionnel de rencontre, les auteurs proposent de combiner différents aspects de l'heuristique et de la praxis psychanalytique, rehaussée par ces deux courants. Empruntant à l'un et à l'autre de ces apports, ils montreront leur pertinence d'articulation concernant la clinique du traumatisme.

Un fragment de prise en charge de Mr A., névrosé traumatique, étayera leur propos.

Mots clés: Jeu, objet a /objet transitionnel, transfert, traumatisme, witz 


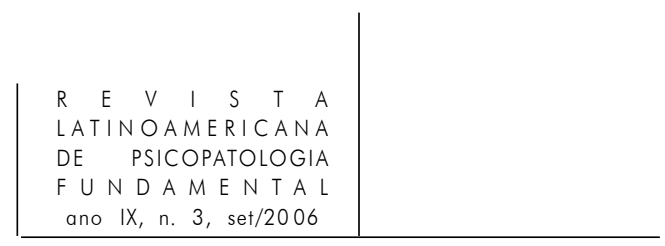

\section{D’une mauvaise à une bonne rencontre}

Quant à la névrose traumatique commune, elle offre deux traits susceptibles de nous servir de guides, à savoir que la surprise, la frayeur semble jouer un rôle de premier ordre dans le déterminisme de cette névrose et que celle-ci paraît incompatible avec l'existence simultanée d’une lésion ou d'une blessure. (Freud, 1919, p. 14)

Cette proposition de Freud à propos de la souffrance du névrosé traumatique, nous oriente d'emblée sur la question topologique, pour ainsi dire spatiale du trauma: hors lieu, hors temps, le trauma freudien se résoudrait plus facilement s'il pouvait se nicher à même le corps, la blessure touchant son bios, l'organique attirant à elle un flux d'investissements censé contrebalancer la déperdition narcissique.

Cette double caractéristique u-topique et atemporelle du traumatisme, est aisément repérable lors de l'instant d'effraction traumatogène et dans son après coup, via les efforts répétés du sujet qui tente de symboliser l'impensable de sa propre perte. ${ }^{1}$ Elle est aussi, cette fois dans son aspect sublimatoire et créatif, la caractéristique d'un espace/temps particulier, celui de l'aire transitionnelle chère à D.W. Winnicott (1971). A la "mauvaise rencontre”, éternellement manquée, de la tuché (Lacan, 1963-64, p. 61-75), qui n'est autre que celle du traumatisme elle-même, se repère la "bonne rencontre" clinique propre au dégagement de cet espace original de l'entre deux. Il donne alors sens à une autre définition possible de la tuché (traduit classiquement par fortune, hasard), du côté de la bonne fortune, d'un évènement qui, tout en échappant au mécanisme de répétition propre à l'automaton, donnera alors sa place au désir inconscient du sujet. ${ }^{2}$

1. Nous faisons là référence au syndrome de répétition avec réminiscence diurne et nocturne de la situation de danger.

2. Tuché et Automaton définissent deux modalités du hasard qui résistent à se réduire aux quatre causes (matérielle, formelle, finale, efficiente) proposées par Aristote. La tuché est une cause par accident qui survient dans les choses susceptibles de choix délibéré mais qui justement ne l'a pas été. "Par exemple, le fait pour un homme de venir sur la place par fortune et d'y rencontrer celui qu'il voulait mais sans qu'il y eut pensé, a pour cause le fait d'avoir voulu se rendre sur la place pour affaires”. Ainsi la tuché donne t-elle sa place au désir inconscient : quelque chose de voulu se réalise sans que le sujet y ait pensé. 


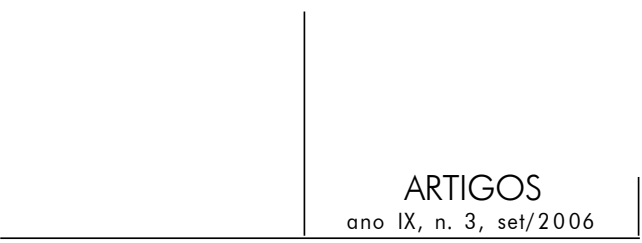

La rencontre amoureuse par exemple, traduit, sur un mode souvent passionnel, traumatogène, parfois négatif et brutal, ${ }^{3}$ cette mauvaise rencontre qui s'avérera in fine convertible en bonne: sans que le sujet n'y ait pensé, il fera sa vie et des enfants avec cet homme ou cette femme... Non anticipable du réel de la tuché "amoureuse".

Or, l'amour, dans le champ psychanalytique, est toujours un amour de transfert, moteur thérapeutique de la cure en tant que le traumatisme positif a justement pour particularité, de transformer les expériences ou impressions traumatiques en produisant

... des efforts pour mettre en œuvre le traumatisme, donc pour remémorer l'expérience oubliée, mieux encore pour la rendre réelle, même si ce ne fut qu'une relation affective antérieure, pour la faire revivre dans une relation analogue à une autre personne. ${ }^{4}$ On réunit ces efforts sous le nom de fixation au traumatisme et contrainte de répétition. (Freud, 1939, p. 163)

Réactualisation du transfert au tenant-lieu de l'Autre donc, le clinicien.

Substituer au lieu du réel (soit un non lieu: impossible de le substantifier de donner une forme, une représentation au trauma; seuls les mots peuvent tenter de traduire son "informe chaotique") le déploiement d'un espace porteur, tel pourrait être l'objectif de la rencontre clinique avec le traumatisé. Cette analogie, entre ces deux espaces, qui n'est surtout pas un amalgame, nous pousse à nous intéresser à la passerelle transféro-transitionnelle entre non-lieu du traumatisme et aire de l'entre-deux. Comment réintroduire du jeu dans la rencontre clinique, à même de mettre de l'huile dans les rouages d'un sujet que le traumatisme a grippé? Tel est l'enjeu de ce présent travail.

\section{Rappel théorique et réminiscences cliniques}

"Est traumatique quelque chose qui lie le sujet à une impossibilité de répondre logiquement ${ }^{5}$ à un événement auquel il est confronté et qui est généralement de l’ordre de la cruauté du monde" (Winter, 1994). La déflagration traumatique doit ici s'entendre dans son lien à la parole, au logos: sous l'excès de jouissance (excès

3. Combien de couples ont-ils débutés leur union par une "mauvaise rencontre" au sein de laquelle l'autre est mal perçu(e), décrié(e) tant pour ce qui concerne son physique (ingrat) que son caractère (imbuvable)?

4. Souligné par nous.

5. Souligné par nous. 


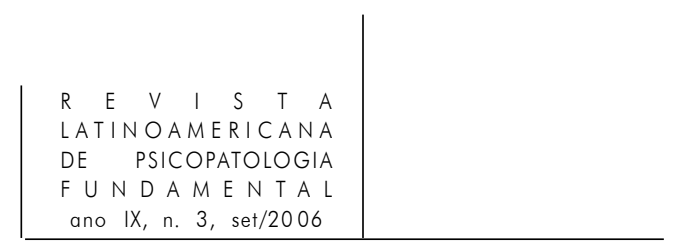

de plaisir et /ou de déplaisir) la parole est mise hors jeu; l'effroi, ça nous la coupe. A sa place, surgira chez le traumatisé une image, un arrêt sur image, réminiscence post-accidentelle de la cruauté du réel, trahissant cette défense de l'imaginaire qui tente stérilement de symboliser l'innommable de la Chose: et se répètent alors les cauchemars de l'impensable évènementiel, qui signent psychopathologiquement parlant, cette sémiologie propre à la névrose traumatique. Le sujet cherche un lieu au trauma, afin de déposer et donc d'oublier, de refouler pour pouvoir dépasser, sa fixation au trauma.

Il est étonnant de relever que, dans sa matérialité, l'objet transitionnel obéit également à ces caractéristiques traumatogènes: hors langage et hors lieu (première possession non-moi qui n'appartient ni à la mère ni à l'enfant), il s'avère non refoulable et non localisable (entre subjectif et objectif, entre réalité interne et externe), si ce n'est bien sûr dans l'espace entre (Winnicott, 1971, p. 13).

\section{Ex-timité et entre-deux}

Il se dégage alors la question du lieu, de la place du traumatisme (l'événement qui frappe le sujet) révélée par notre phrase introductive qui, en provenance de l'extérieur envahit de tous ses effets l'intérieur de l'être, pour se concrétiser en trauma (l'effet d'un tel événement chez le sujet). Il en va de même pour l'objet transitionnel, à la fois trouvé (c'est-à-dire réellement présent, voire présenté ou plutôt présentifié à l'infans (object presenting mais aussi handling et holding - Winnicott, 1971, p. 154) et créé (au fondement de sa réalité psychique interne).

Le paradoxe, nous dit Winnicott, ne doit pas être résolu. Lacan a également pensé le trauma, comme le réel (et son corollaire de jouissance) de manière paradoxale, ce que révèle le néologisme d'“ex-timité" (de la Chose) (Lacan, 196869, séances du 12 et 26 mars 1969).

Ex-time renvoie simultanément à l'idée que la jouissance (traumatogène car désubjectivante) est à la fois ce qu'il y a de plus étranger à l'humain (ex), et donc ce dont il doit se protéger par toutes les voies (symptomatiques) possibles, mais aussi de plus familier, de plus intime à chacun. En matière d'expérience (procès structurants) ou d'épreuve psychique (traumas déstructurants), il nous semble plutôt limité d'opposer, au sein de la fiction qu'est toute théorie, ${ }^{6}$ une réalité interne à une réalité externe - ce que la théorisation des "enveloppes psychiques”, du 
“Moi-peau” propre à l'école d'Anzieu nous paraît valider. Il s’agira alors de condenser l'espace psychique en un lieu paradoxal, ni à l'extérieur, ni à l'intérieur mais "entre", (Winnicott) ou de le confondre en un lieu double, et à l'extérieur, et à l'intérieur (Lacan).

\section{Espace potentiel et espace transférentiel}

Cette localisation paradoxale ou impossible nous intéresse au plus haut point, non pas tant de par l'analogie qui trouve là, dans notre propos, sa limite heuristique, que dans la création de cette autre scène, celle du transfert, d'où la psychanalyse tire sa pertinence praxique.

En effet, l'entreprise de Winnicott consiste à trouver une place au jeu, à dégager à partir de lui un espace qui ne pourra être contesté au sujet. Ce lieu potentiel sera plus tard, certes, celui des activités (sublimatoires) créatrices, de la fantaisie, de l'imagination, bref, de la culture et des activités artistiques, mais également, et surtout, celui de l'analyse et du transfert (Mannoni, 1990, p. 40). C'est dans cette part de jeu (et de contre-jeu maternel) que le sujet façonne son "premier espace potentiel” préfiguration de tous les futurs "playgrounds" ultérieurs. Que le jeu vienne à manquer et le bébé doit se fabriquer un faux self au risque d'en passer par "la détresse impensable" (ibid., p. 41).

Or, détresse et trauma ne font qu'un: l'“Hiflosigkeit” freudien est traduisible par "trauma": l'élément traumatique n'est autre que la détresse ${ }^{8}$ elle-même, soit cette exposition à la non-satisfaction des besoins primaires, qui implique la mort, et ce sans recours possible à l'Autre. Winnicott rejoint alors Ferenczi dans la conceptualisation du traumatisme, pensée alors comme résultante d'une situation de détresse vécue sans réponse de la part de l’objet (maternel), ce qui a pour effet de procéder à une atteinte précoce du moi, et à une véritable blessure narcissique faite à l'infans (Bokanowski, 1999, p. 71). Winnicott semble alors revenir à Freud, qui écrit à l'occasion d'une correspondance à Jones (lettre du 18 février 1919): “A la base de chaque cas de névrose de transfert se découvre une névrose traumatique”. Tout comme il paraît se réarticuler aux travaux de Lacan concernant l'objet pulsionnel voix: ce qui est traumatique dans la transmission du legs langagier à l'infans, c'est que le signifiant (du Nom du Père) vient de l'Autre, le (pré)-sujet se positionnant alors en fonction de la qualité d'une telle transmission;

7. Littéralement “cour de récréation”.

8. “Nommons traumatique une telle situation de détresse...” Freud, 1926. Cité par Toboul, 2003. 


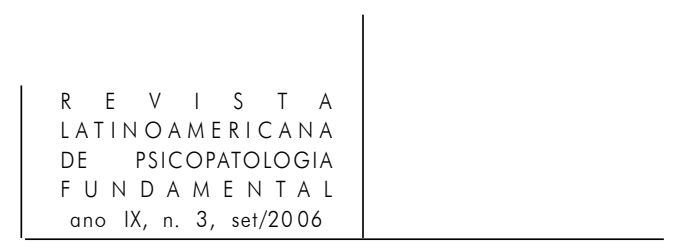

si l'on peut fermer les yeux sur l'irregardable, le nourrisson n'est pas en capacité de se boucher les oreilles afin de se protéger de l'inaudible. ${ }^{9}$

Ces correspondances, ce transfert d'idées et de conceptualisations, précisent leur valeur de filiation et/ou de transmission heuristique: à suivre Winnicott, il ne resterait au sujet qu'une alternative d'exclusion/inclusion, si celui-ci n'est pas arrivé à constituer cette aire transitionnelle. Est ce à dire que le sujet n'a plus alors d'autres choix qu'un maniement répétitif à l'identique de l'événement?

Ces différentes passerelles notionnelles devront pourtant s'arrêter là; en repartant de la phrase susdite de Freud, nous instaurerons une limite de conceptualisation du trauma entre l'approche freudienne et celle du psychanalyste anglais.

\section{Winnicott sans Freud}

Le mécanisme de répétition (de transfert ${ }^{10}$ ), se conçoit pour Winnicott, du point de vue de la maîtrise de la situation pénible dans laquelle se trouve l'enfant lors du départ de la mère, ou bien lorsque celle-ci ne reflète rien d'autre qu'une vacuité dépressive. ${ }^{11}$ Pour Freud, dans ses derniers écrits sur la question du traumatisme (Freud, 1939), il trouve son point d'origine dans le trauma positif, qui donne toute son impulsion à l'effort pour mettre en œuvre le traumatisme, donc pour remémorer l'expérience oubliée auprès d'une autre personne. Quant au trauma négatif, il reste également central dans le mécanisme de répétition, en tant qu'élément mythique nécessaire à la métapsychologie freudienne: si ce dernier tend au but opposé du trauma positif, c'est parce qu'il empêche les activités de répétition, de remémoration, et d'élaboration du trauma, en s’avérant “horstransfert". Ne s'agit-il pas là de la "fiction"12 propre au refoulement primaire par exemple (par définition non re-mémorable)? A partir de lui, véritable noyau centrifuge aux représentations rejetées secondairement, l’opération du refoulement

9. Lacan dans le séminaire XI nous rappelle que "Les oreilles sont dans le champ de l'inconscient les seuls orifices qui ne puissent se fermer”, p. 178.

10. Il s'agirait là alors plus que d'un transfert de désir, d'un transfert de besoins (narcissiques primaires) chez ce dernier. Se reporter aux travaux de Winnicott sur "De la régression considérée comme une thérapie”, 1954.

11. Lire à ce propos les travaux de Winnicott sur le visage de la mère, en particulier "Le rôle de miroir de la mère et de la famille dans le développement de l'enfant”, 1971, p. 153-62.

12. Nous faisons ici référence à l'ouvrage de M. Mannoni “la théorie comme fiction”. 
tient debout d'un point de vue théorique. Cette opération ne se résume t-elle pas à ce reste indépassable d'une mise en forme du réel auquel Lacan donnera le nom de structure? Dés lors nous concevons que ce qui distingue la psychanalyse des autres approches psychologisantes de l'humain, c'est que pour elle et pour elle seule, le sujet advient du trauma (Assoun, 1999, p. 10). C'est là, nous semble t-il, le distinguo princeps entre Freud et Winnicott, distinguo confirmé par Freud lorsqu'il écrit: "On peut tout de même à juste titre caractériser le refoulement, qui est à la base de toute névrose, comme une réaction à un traumatisme, comme une névrose traumatique élémentaire" (Freud, 1919, p. 247).

Au-delà de cette distinction essentielle, subsiste néanmoins une correspondance majeure, celle de la portée de la dimension langagière ${ }^{13}$ (Freud postulera finalement une transmission du trauma de et par le langage -Freud, 1939) et du principe d'altérité, tous deux aux fondements du transfert et de la "transitionnalité objectale".

\section{Autre maternel et langage}

La tuché propre à l'amour, celle du nourrisson à l'égard de la mère suffisamment bonne, ${ }^{14}$ ou de l'amant à l'aimé, est susceptible de se transformer en "bonne fortune" dans la mesure où il $\mathrm{y}$ a de l'A(a)utre, condition sine qua non d'une rencontre et d'un lien objectal. Mieux, pour ce qui concerne le traumatisme, cet autre doit être rétabli à sa juste place, symbolique, alors même qu'en cas de mauvaise rencontre, l'effroi, la détresse, évacuent toute dimension d'altérité. ${ }^{15}$

La rencontre clinique, rencontre de - soi au travers d'un rapport nonsymétrique de - l’altérité, tire sa pertinence et son efficacité thérapeutique du

13. Et ce, malgré la relégation au second plan de l'aspect langagier, tout au long de l'œuvre de Winnicott.

14. Winnicott pense le passage de la bonne mère à la mère suffisamment bonne, comme la capacité de la mère à percevoir chez son bébé, une possible réaction de colère au lieu d'être traumatisé par le défaut d'adaptation, l'absence de réponse de cette dernière (Winnicott, 1967, p. 29). Or, la colère, est un affect qui, comme tout affect, nécessite son objet, soit une adresse à la dite colère. Winnicott traite ainsi de la relation d'objet, dans sa distinction du sujet, corroborant ainsi nos propos quant à l'importance de la place, de l'Autre dans le développement de l'être (... ou dirions-nous, de l'avènement du sujet).

15. L'Autre a trahi le sujet au moment du chaos traumatogène accidentel: ni la Loi symbolique du signifiant, ni le pouvoir de mise en forme de l'Imaginaire ne peuvent "supporter" la charge, ni participer à la bonne tenue du sujet dans le monde. Se reporter à Cabassut et Ham, 2004. 


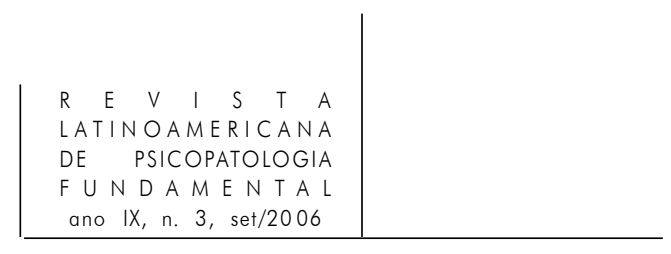

rétablissement de l'Autre, dont le clinicien occupe, dans le transfert, le tenant-lieu. Importance de la place donc, de l'adresse dans la dynamique du transfert, qui nous autorise le lien entre altérité et langage. En effet, le transfert, dans son acception lacanienne est un transfert non d'affects, de sentiments, ou de pensées (au sens télékinésique du terme), mais de signifiants. C'est dire le rapport étroit entre transfert et langage.

Et l'objet transitionnel est également affaire de langage. ${ }^{16}$ Celui-ci n'a pas pour visée essentielle, comme la bobine de fil du célèbre jeu du "Fort/Da" (Freud, 1919, p. 13-20), d'incarner cet effort de maîtrise (du départ de la mère), de possession (d'un objet dont on ne peut se défaire faute de se délier, de s'effondrer). Son efficience, il la tire du symbolique langagier (Mannoni, 1990, p. 42): le nom de phénomènes transitionnels peut être attribué à un bouchon de laine, un coin de couverture ou d'édredon, un mot, ${ }^{17}$ une mélodie, ou encore un geste habituel, ${ }^{18}$ bref autant d'objets ou phénomènes qui acquierent une importance vitale pour l'enfant lors de l'endormissement (Winnicott, 1971, p. 11). Dans le même ordre d'idée, Winnicott précise:

Dès que le petit enfant commence à utiliser des sons organisés (mam, ta, da), un “mot” peut apparaître qui désignera l'objet transitionnel. Le nom donné par le petit enfant à ces premiers objets est souvent significatif; il témoigne généralement d'une incorporation partielle d'un mot utilisé par les adultes. Ainsi l’objet peut être désigné par le mot "baa" où se retrouve le b de "baby" ou de

16. Nous n’assimilons pas pour autant ces trois modes de communication dégagés par Winnicott: celui de la communication silencieuse propre au self central; un mode explicite, indirect, caractéristique de l'emploi du langage; et enfin, un mode intermédiaire, qui du jeu passe à la vie culturelle (se reporter à Green, 1990, p. 7-9). Cependant, notre démarche d'inspiration freudo-lacanienne, vise à redonner au langage, en tant que celui-ci est contaminé par la parole, langue de l'infantile, la place de vecteur princeps dans l'avènement du sujet. Prolongement donc, d'une théorie d'inspiration développementale qui néglige le rôle du langage, en tant que celui-ci est travaillé par la pulsion (soit la sexualité infantile, la jouissance) comme la dimension paternelle. La discrétion sur l'effet de langage, la place du père, le rôle de la pulsion (au profit de la communication, de la mère, du lien de continuité) forme autant de points aveugles dans l'approche winnicottienne.

17. Souligné par nous.

18. Nous retrouvons ici la dimension signifiante langagière, sur laquelle D.W. Winnicott s'avère si discret, et que Laplanche localise dans ces signifiants verbaux, voire comportementaux inconscients imprégnés de significations sexuelles que l'adulte propose à l'enfant (Laplanche, 1987, p. 630). La préoccupation maternelle précoce peut donc se définir comme l'introduction du sexuel dans “un premier corps de signifiant” (Lacan, 1955-56, p. 171) chez l’infans. 


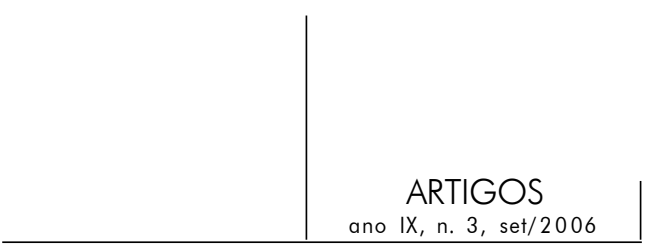

“bear”; Parfois, il n’y a pas d’objet transitionnel autre que la mère elle-même. $(\text { p. } 12)^{19}$

Trauma (dans son lien au logos), transfert et espace transitionnel (qui nécessitent l'Autre du “stock lexical”) se retrouvent ainsi noués. Ils nous orientent vers un certain type de praxis clinique auprès du traumatisé:

- Retour de l’A (a)utre, qui prend alors les traits du clinicien

- Place pour le jeu, à même de redéfinir le je

- Réintroduction du jeu transférentiel ${ }^{20}$

- Primat d'une clinique langagière

Ces divers points nous semblent venir corroborer nos observations cliniques. Lors d'un précédent travail, ${ }^{21}$ nous avons pu en effet mettre en lumière ce que serait le cheminement transférentiel prototypique du traumatisé:

- Demande spontanée de prise en charge, de rencontre adressée à l’ (A) autreclinicien

- Expression massive de la plainte et de la douleur (physiologique)

- Associations langagières riches et prolifiques (propre au sujet en "état de crise”)

- Positionnement du clinicien en place d'“Autre supposé savoir” (Didier-Weill, 1995, p. 145), comment rabaisser sa détresse.

Jeu langagier et jeu transférentiel semblent ici s’associer dans l'avènement d'un espace transféro-transitionnel de rencontre. Co-implication et co-émergence du transfert et du jeu qui ne font qu'un, dans la lutte qu'ils livrent à la détresse du traumatisé. Dès lors, nous comprenons aisément que si le jeu est thérapeutique par lui-même, alors le transfert advient également en tant que moteur de la cure, dans la mesure où il introduit du jeu: le transfert est ré-création du rapport à l'Autre. Dès lors, nous avancerons en empruntant à la logique des paradoxes chère à Winnicott, que le lieu du transfert n’est autre que la réactualisation de ce lieu

19. Le “doudou” communément interprété en guise d’objet transitionnel nous paraît à ce titre se rapprocher plus de la bobine, objet contra-phobique dont la possession protège illusoirement de l'absence de la mère, que s'instaurer en substitut de transition (le substitut étant justement autre chose qu'un représentant ou qu'un signifiant; Mannoni, 1990, p. 42).

20. Puisque toute névrose “ordinaire” est traumatique, elle n’atteint la dignité d’une névrose de transfert qu'en situation de cure (Ham, 2003, p. 81).

21. Se reporter à Cabassut, Jacques et Ham Mohammed. Les victimes du temps de dire. A propos des cellules d'écoutes médico-psychologiques en urgence. Filigrane, Montréal. A paraître courant 2005. 


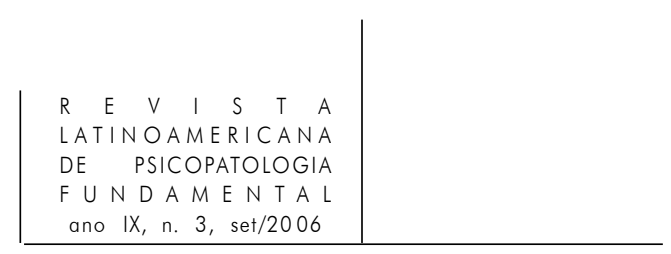

u-topique qu'est l'aire transitionnelle... et inversement: le grand véhicule du transfert crée la transition indispensable au transport de signifiants. Conjonction, plutôt que confusion, du lieu de la transitionnalité et du transfert. Il s'agit là d'un seul et même espace que nous qualifierons de transféro-transitionnel. Nous devons, à présent, en cerner les implications théoriques et cliniques, nous inspirer de cette re-trouvaille qui est aussi une création (objet trouvé-crée), afin de l'injecter dans notre praxis: l'analyste guérit moins par ce qu'il dit et fait que par ce qu'il est, nous instruit Lacan (1958, p. 587).

\section{Objet a et objet transitionnel}

“Ça (l'objet a) s'apparente à la logique, c'est-à-dire que ça le rend opérant dans le réel au titre de l'objet dont justement il n’y a pas d'idée (...)” (Lacan, 1975, p. 182). Winnicott proposera également un objet dont on n’a pas idée, qui ne peut se refouler, l'objet transitionnel. On sait que Lacan s'en est inspiré afin d'inventer son objet, le “a” (Porge, 2000, p. 175), qu'il va placer au centre du nouage borroméen, supporté par l’Imaginaire, le Symbolique, le Réel et le Nom 396 du père (quatrième anneau implicite au fondement du maintien de la structure). Objet cause du désir, dans la mesure où il est maintenu à sa place centrale, l'objet “a” assure également le sujet, en supportant sa course désirante. C'est pourquoi, les trois faces de l'objet “a”, qu’A. Didier-Weill désigne par les dimensions d'Inouï, d'Invisibilité, et d'Immatérialité (Didier-Weill, 1995), déterminent la bonne tenue au monde du sujet. La topologie lacanienne rejoint ici la spatialité Winnicottienne: à l'entrecroisement des quatre (anneaux) au lieu de l'entre-deux. Ainsi, ce sur quoi nous voudrions insister ici, c'est sur l'analogie entre ces trois dimensions symboligènes, et celles de l'espace potentiel:

- L'Inouï $(\mathrm{R} / \mathrm{S})^{22}$ est cette dimension mélodique, musicale, qui habite et transcende le signifiant, la parole (tel que les phonèmes winnicottien, " $b$ ", "baa", que Lacan aurait placé du côté de la lalangue, nous le révèlent). Elle est véhiculée par la voix de l'Autre maternel, et transmise à l'infans. Or, la musique se déploie sur ce terrain neutre, ni en provenance de l'extérieur ni dans une écoute intérieure, ce qui la démarque de tout caractère dommageable, envahissant, menaçant ou traumatique... Quant à la décompensation psychotique, comme dans le cas du président Schreber (Freud, 1911, p. 263-324), elle “s'entend” à partir d'un appel 


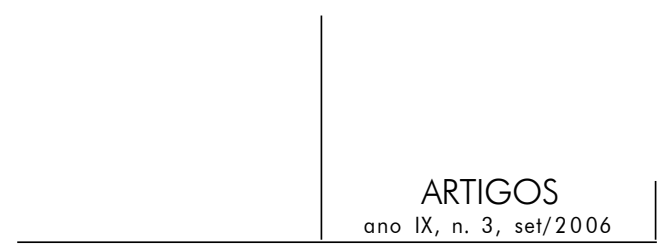

du signifiant (du Nom du père) qui convoque (et non qui invoque, tel l'élément musical) le sujet à accéder à une place qu'il ne peut occuper.

- Tout comme l'objet a, non-spéculaire, l’objet winnicottien obéit à cette dimension d'Invisibilité. Même le visage de la mère, cher à Winnicott, ne fait pas qu'image, d'un point de vue perceptif ou représentationnel, puisqu'il s'avère être le précurseur du miroir (Winnicott, 1971, p. 153). L’objet transitionnel est désigné par une forme (pré)symbolique (Mannoni, 1989, p. 42), ${ }^{23}$ tout comme l'Invisible (S/I), ${ }^{24}$ “cet au-delà de l'image” qu'est le symbolique (Didier-Weill, 1998, p. 27), dans la mesure où elle reste sous son ascendant. Le jeu s'inscrit du côté de la maîtrise symbolique (et non pas du côté de la crispation possessive imaginaire de l'objet).

- L’Immatériel n'est pas à confondre avec le non matériel. Certes, il s'agit d'une “existence effective”, matérielle, de l'objet (sein, bout de couverture etc.); mais, "l'objet transitionnel est justement ce que nous percevons du voyage ${ }^{25}$ qui marque la progression de l'enfant vers l'expérience vécue” (Winnicott, 1971, p. 14). L'immatériel ( $\mathrm{I} / \mathrm{R})^{26}$ est cet effet de la rencontre du signifiant, qui va arracher le corps à sa matérialité pesante réelle, en octroyant au réel du corps sa part d'Inouï et d'Invisibilité. L'Immatériel peut donc se concevoir comme ce "voyage", cette bonne transmission, cette circulation "suffisamment bonne" (de et dans la chaîne signifiante) du signifiant, qui investit le corps et assure la bonne tenue au monde du sujet.

Objet a et objet transitionnel co-émergent donc simultanément, afin de désigner le sujet: “Le sujet inconscient n'a pas de sexe ni de prétentions. Son activité c'est le jeu. (...) Le sérieux c'est l'affaire du moi. Donc parfois du faux self” (Mannoni, 1989, p. 45). Lorsque la part subjectivante du jeu est annihilée, comme en cas de trauma, alors le sujet perd ses dimensions d'Inouï, d'Invisibilité et d'Immatérialité; à la place, en surgissent trois autres que nous avons précédemment isolées et nommées (Cabassut, 2002, p. 199-200) dans leur rapatriement dans le champ du réel: l'Inouï se transforme en Innommable, l'Invisible en Irregardable, l'Immatérialité du corps en Inconsistance.

23. L'image ne vaut qu'à être nommée par l'Autre maternel, comme le procès du miroir nous le rappelle.

24. Lire une négativation de l'Imaginaire par le Symbolique (S/I).

25. Souligné par nous.

26. Lire une négativation du Réel par l’Imaginaire. 


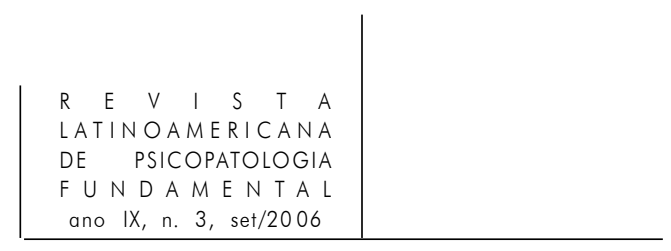

\section{Le sujet transitionnel}

Ce point de ralliement théorique qu'est le sujet de l'inconscient, nous autorise à poursuivre notre correspondance entre espace transitionnel et jeu transférentiel, pour avancer la proposition suivante: instaurer le transfert consiste donc à introduire du jeu, à même de faire circuler des signes, des signifiants entre et auprès des deux protagonistes. Rencontrer le sujet, restaurer ses dimensions d'Invisibilité, d'Immatérialité et d'Inouï au sein de la rencontre, c'est remettre en jeu cet espace potentiel de et dans la rencontre.

Le trauma empêche sur un plan symptomatique la bonne circulation de la pensée, puisque "penser n'est rien d'autre que manipuler des signes” (Gori, 1996, p. 10). L'analyste qui délivre une interprétation - énigmatique - relance la polysémie signifiante, en ouvrant sur un redéploiement possible et infini de sens. Il ne s'agit pas tant, alors, de suturer de manière univoque, d'arrêter le sens (de circulation signifiante) que de le complexifier, d'en maintenir vivant la dynamique, son jeu inter et intra représentationnel. En effet, s'“Il n' y a de résistance que de l'analyste" (Lacan, séance du 19 mai 1955) c'est dans la mesure où son écoute se fige sur un élément qui parasite le flux associatif, arrête l'élan circulatoire.

Le maniement du transfert consiste donc, d'abord pour celui qui est en place de tenant-lieu de l'Autre, à sauvegarder sa propre capacité "transitionnelle" à jouer, à faire exister cet espace potentiel. Les entretiens préliminaires à la cure par exemple, consistent à évaluer si ça passe entre moi et l'autre. ${ }^{27}$ Les réaliser dans une optique de diagnostic structurel, ferme la rencontre du côté d'un impossible, celui de prendre un psychotique en analyse par exemple. Si l'hystérique ne l'est que sous transfert (Ham, 2003, p. 118-9), c'est justement parce que, quelle que soit sa psychopathologie, on attribue au sujet cette capacité intrinsèque et irréductible à jouer - se jouer parfois de l'A(a)utre - du transfert. L'utilisation de test, la situation de testing ${ }^{28}$ elle-même, permettent d'illustrer au mieux la création de cet espace transféro-transitionnel.

Mais, notre propos dépasse ici le simple cadre technique: c'est le style même de l'analyste, du clinicien (et donc de la cure, de la rencontre clinique) qui s'élabore en fonction d'une telle potentialité: le sujet du transfert et celui de la

27. Et parfois ça ne passe pas, comme le souligne J. P. Winter, qui défend l’idée que l'analyste ne peut pas garantir une qualité d'écoute sous transfert avec n’importe qui. A ce titre, il doit s'autoriser à ne pas prendre le sujet en analyse (Winter, Jean-Pierre. Séminaire Montpelliérain du "Coût freudien”, 2003. Non publié).

28. Se reporter en la matière aux travaux de P. Lekeuche, 1989. 


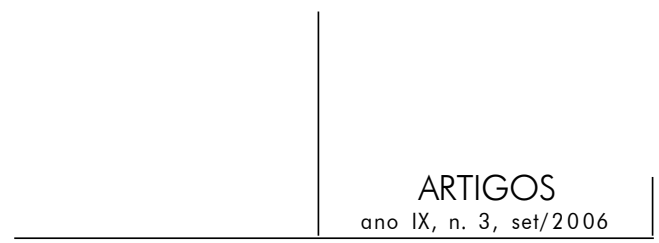

transitionnalité, le je et le jeu, ne font qu’un, même si la place dans le transfert et l’objet de la transition ne sont ni identiques, ni équivalents.

\section{Le sujet transférentiel}

Pour Lacan, le rapport à l’objet s’inscrit du côté de la perte, du déchet. L’objet représente cette partie absente du corps de la mère, à jamais perdue. Il est marqué du sceau de la séparation irrémédiable. Il s’agit alors du point de vue de la pratique clinique “d'exaspérer la distance”, celle qui consiste à mesurer l’impossibilité où se trouve tout désir d'accéder à sa satisfaction (Clément, 1990, p. 66-7).

Selon Winnicott, le sujet "se fait” son objet, afin de réaliser un passage (de la dépendance vers la non-dépendance) dans le sens de la préservation d'une continuité (alors menacée par la rupture à l'Autre maternel), un objet qui va nous accompagner toute notre vie durant, au fondement du sentiment de soi, et qui deviendra un espace incontestable. Il faudra alors, cliniquement parlant, contacter ce lieu afin que le sujet s' " $y$ ” retrouve.

D’un côté, la limite, la scansion, la coupure, la frustration et/ou la castration... L'analyste "fait" le mort. Ses interventions sont marquées par le poids d'un silence symboligène et d'une attitude de neutralité bienveillante. La dérive consiste alors, pour le clinicien, à se crisper sur une position paternelle figée, voire morbide, tant elle en devient froidement impersonnelle, véritable incarnation d'une place vide.

De l'autre, la dépendance, la régression, le rétablissement de la communication silencieuse, le recouvrement du faux-self (à la place de la frustration et de l'évaluation de la distance à l’objet perdu)... L'analyste est vulnérable (à condition de ne pas quitter son rôle professionnel nous indique Winnicott). ${ }^{29}$ Ainsi la psychothérapie, du fait de la plus grande liberté qui s’y déploie, intéresse t-elle plus ce dernier que la cure type (Mannoni, 1990, p. 43-4). L'excès d'un tel modèle est de déboucher sur une position maternelle, voire maternante (à laquelle Winnicott n’a pas échappé), réveillant des mouvements identificatoires mous et moïques, voire inconsistants, à la "bonne mère".

Comment, dès lors, dans le maniement du transfert et de l'heuristique analytique, concilier ces deux positions contradictoires?

29. Vulnérable signifie "exposé sans protection”, sans avoir recours de manière défensive à la théorie, et ce afin d’inventer sa pratique de manière créative. Cité par O. Mannoni, 1990. 


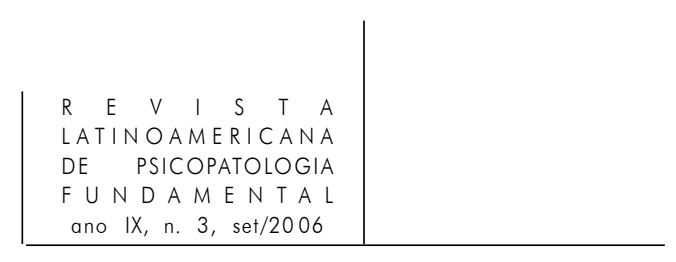

Le transfert, rappelons-le, n'est autre que ce transport amoureux de signifiants. Il nécessite donc un espace particulier, afin que les signes circulent, celui de l'aire transitionnelle, grand espace du transfert. Sans lui, les mouvements transféro-contre-transférentiels sont à l'arrêt. Avec lui se fabrique un espace de jeu, de médiation contactuelle (au sens Szondien du terme), espace ni fusionnel, ni spéculaire, dans lequel chacun se vit comme à la fois séparé et relié à l'autre. (Lekeuche, 1989, p. 328)

Cependant, un tel espace n'est rien sans l'échange langagier signifiant. Pour être "touché" par la parole, l'espace intermédiaire doit exister. Mais celui-ci ne se crée que dans la (re)-trouvaille du langage, qui implique d'emblée la dimension d'altérité (je parle toujours à un $\mathrm{A}(\mathrm{a}) \mathrm{utre}$ ) et la nécessaire "transposition" et

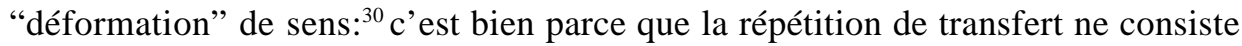
pas en une reproduction à l'identique, que quelque chose de nouveau peut advenir dans la rencontre, ${ }^{31}$ quelque chose de "tychique" (Lacan, 1963-64, p. 90), où le sujet se surprend à dire ce qu'il n’attendait pas (Lekeuche, 1989, p. 327). Tel est l'enjeu de la prise en charge clinique du traumatisé: là où le sujet répète stérilement le signe-symptôme ${ }^{32}$ de sa souffrance post-traumatique, il faudra réintroduire du jeu et élever le signe à la dignité du signifiant.

Réintroduire du jeu au sens mécanique du terme, là où le trauma a enkysté le traumatisé; et ce par le biais du transfert, inséparable du langage, dans la mesure où il en produit une traduction et donc une trahison de sens (ibid., p. 326). Bref, sous transfert, le signifiant est une création, qui se déploie dans un espace potentiel au sein duquel le sujet (clinicien et/ou traumatisé, analyste comme analysant) peut se surprendre. Telle est d'ailleurs, la définition du signifiant dans sa distinction au signe (symptôme): le premier ne signifie rien pour personne (ni pour l'analyste ni pour l'analysant), a contrario du signe qui signifie quelque chose pour quelqu'un. ${ }^{33}$ Le signifiant, à ce titre, n’appartient ni à l'espace extérieur (même si dans sa forme interprétative il provient du dehors, de la place de

30. Freud utilise le terme d'Entsellung à propos du transfert qui signifie "transposition" et “déformation”, c’est à dire trahison de sens. Se reporter à Lekeuche, 1989, p. 326.

31. Pour Lacan, c'est à la fin de l'analyse que le sujet en sort après avoir construit son "sinthome", soit cette solution para-névrotique ou para-psychotique qui pallie au défaut de transmission du Nom du Père. Se reporter à Julien, 2000, p. 67-91.

32. Il peut s'agir d'un bruit (consécutif à une explosion), d'une pensée (celle du je suis mort qui a pu traverser le sujet au moment traumatique), d’une odeur (qui a imprégné le sujet), d’une vision (d'effroi, celle concernant ses lésions corporelles par exemple, métaphores hideuses de l'irregardable de sa propre perte...)

33. Se reporter à Vives, J-M, 1999. 


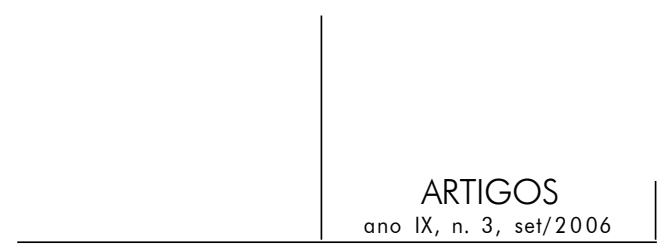

l'analyste), ni à l'espace interne, psychique du patient. Il échoue là, dans cet espace intermédiaire, à l'entre-deux. Dès lors, au-delà de la justesse de l'interprétation, c'est bien la valeur anti-dépressive du dit espace qui est primordiale; ce que confirmerait Winnicott pour qui la valeur symbolique du phénomène transitionnel est secondaire, eu égard à sa qualité de présence (ibid., p. 328). La parole interprétative donnée, trouve alors toute sa "portée", celle de se constituer non pas tant en parole de révélation, qu'en parole de médiation... transférentielle. Il y a de l'A(u)tre dans le jeu transférentiel, un autre que l'on peut alors rencontrer "comme par hasard", et qui peut nous surprendre et nous suspendre, au lieu de l'effroi propre à la mauvaise rencontre de la tuché.

\section{L'interprétation qui prend valeur de mot d'esprit}

Mr A. est un jeune adulte grièvement brûlé, suite à l'explosion d'une bouteille de gaz. Il vient consulter à cause d'une souffrance post-traumatique cauchemardesque massive: l'odeur (de peau cramée) et la vision de sa peau qui fond sous l'effet de la chaleur, hantent ses nuits. Dans son parcours de vie, il a auparavant fait de la prison et par deux fois s'est retrouvé en garde à vue. A l'occasion d'une rencontre, nous lui demandons d'associer sur cette odeur: "une odeur de poulet que l'on crame" dira-t-il. Nous lui signalerons alors que poulet, dans le langage familier, c'est aussi le flic, celui qu'il a été amené à rencontrer plusieurs fois. Après un temps de vacillement, il sourit et dit: "Et bien avec vous on parle des choses de façon bizarre...”. A la suite de cette séance, le brasier de souffrance symptomatique à contenu événementiel, plaintif et dépressif s'est peu à peu éteint rallumant par la même une dimension du dire qui, par son adresse, ses hésitations, ses failles, ses interpellations, ses reprises et ses ratés, inscrivait dynamiquement l'événement du côté de l'oubli, dans une tentative de maîtrise de l'effroi par la parole.

Faire le jeu de l'Autre ou avec l'Autre?

Jeux de mots donc, et pouvoir (partiel) du symbolique face à la cruauté du réel. Tel est la force de l'interprétation signifiante qui prend valeur de trait d'esprit. Le Witz ${ }^{34}$ (mot d'esprit) est en effet, Freud (1905) nous ayant instruit de son lien 


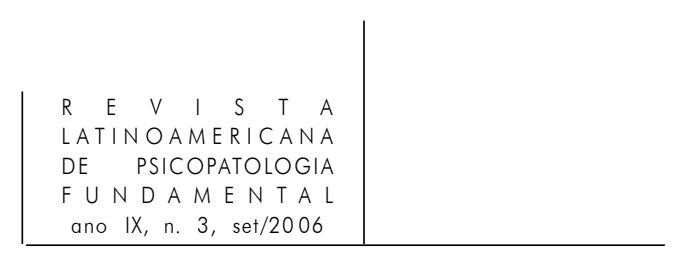

à l'inconscient, au principe du sujet. Procédé de condensation ${ }^{35}$ métaphorique ${ }^{36} \mathrm{du}$ mot d'esprit, également présent dans le rêve, qui expulse le signifié traumatique (odeur, et mal vision) tout en le signifiant implicitement. Le Witz travaille donc à l'économie. Il réalise un plaisir spécifique tout en épargnant une dépense, dépense d'inhibition (empêchement d'agir, en l'occurrence pour ce qui concerne Mr A., de dormir) ou de répression. Il nécessite l’A(a)utre, faute de se réduire alors au private joke, celui utilisé abondamment dans son travail d'écriture par Joyce, et qui, comme le souligne Lacan, a la particularité "de ne faire rire que lui” (Lacan, 1975-76). Un Autre, rappelons-le, qui a abandonné le sujet au moment du trauma, et que ce dernier peut retrouver à sa place, dans le transfert symbolique, le transport de signifiants, en tant que garant de l'ordre symbolique langagier comme de sa destinée de sujet. Bref, nous effectuons par le truchement du Witz une forme de retour sur la fonction du trauma positif freudien, qui transforme le trauma négatif propre à la tuché accidentelle, en trauma positif au sein d'une "bonne rencontre" clinique: la jouissance du Witz peut être abordée comme un réel producteur d'effets, dans la mesure où le trait d'esprit est à même de s'inscrire au sein de la répétition transférentielle (propre au trauma positif). Le Witz ne renvoie pas à une perte sèche du réel, cet impossible, et ce, malgré les tentatives réitérées, mais stériles du sujet (trauma négatif), à symboliser ce qui n’a pu l'être (rêves cauchemardesques).

Cet autre, dans l'humour généré par le Witz, augmente mon propre plaisir en retour, ce qui a pour effet de transformer, par voie rétroactive, le plaisir en jouissance: je jouis de mon propre plaisir sous l'effet de cette rétroaction (Assoun, 1999, p. 176-90). Le Witz représente donc une modalité sublimée de l' "Au-delà du principe de plaisir”: le sujet recouvre le narcissisme détérioré par le trauma, au travers de la toute puissance des mots, ce qui lui permet de gagner sinon la guerre tout au moins une bataille sur la mort. Le sérieux de l'humour se dresse contre la gravité de la mort, dont Mr A. a fait la "mauvaise rencontre" lors du trauma. Il est un remède narcissique à la dépression, à la déception, liées à la perte de toute puissance post-traumatique.

35. Le processus de condensation permet de transformer un contenu latent en contenu manifeste, à l’instar du travail du rêve.

36. Dont l'étymologie nous renvoie au grec “métaphora”, qui signifie transport. 


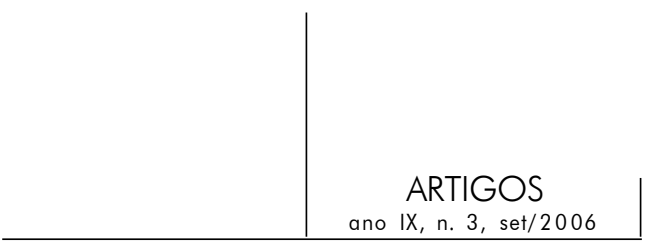

Du trauma dénarcissisant au Witz renarcissisant

Ce point nous intéresse particulièrement en cas de trauma. En effet, toute interprétation, pour celui qui en fait les frais, est une blessure narcissique. Le risque de blesser le sujet est donc accru chez le traumatisé, dans la mesure où celui-ci est déjà en déficit narcissique. La "technique interprétative" opérante chez le névrosé traumatique, doit donc s’élaborer à partir de cet aménagement par le jeu langagier qui permet l'obtention de ce gain économique propre à l'humour, soit l'apport de l'effet de surprise (observable dans le commentaire de Mr A.) tout en réduisant la part de déconstruction narcissique qui lui est inhérente. A ce titre, la surprise constatée lors de la rencontre du signifiant symbolique, et la sidération qu'il engendre, se distinguent de l'effroi désubjectivant et traumatogène issu de la "mauvaise rencontre" du réel. La différence princeps tient en ceci: pour le premier, la sidération peut laisser place à la dé-sidération (à l'image de la parole finale de Mr A.); chez le second, la sidération perdure au détriment d'une désidération somme toute "irréelisable”. La "Witz-interprétration" ne se constitue donc pas simplement en "blessure narcissique" pour le sujet: ce qu'il entend est promesse symbolique d'advenir en cette terre d'accueil inconnue (mais non méconnue) que constituent l'Invisible, l'Inouï, l'Immatériel de son "être psychique” (Didier-Weill, 1995). Nous pouvons lire en filigrane, la pratique de coupure chère à l'approche lacanienne, une coupure signifiante recevable narcissiquement pour le sujet. L'interprétation en tant que don symbolique de parole fait entendre l'Inouï, par le truchement de l’inédit d'une parole.

\section{La voix de l’amour de transfert}

Autrement dit, ce que le sujet entend au-delà du procès signifiant, c'est une musique, particulière, celle du jeu, au fondement du je. C'est cette musique qui rend la chose entendue, acceptable et appropriable par lui. La mélodie musicale dans son lien à la pulsion invocante, à l'objet pulsionnel voix, permet ainsi d'accéder à un point de jouissance sur un mode sublimatoire, ${ }^{37}$ et non plus invasif, comme lors du trauma. Nous sommes bien alors dans cet espace mythique et

37. A ce titre A. Didier-Weill parle du "point bleu” en tant que ce point de musicalité idéal que le musicien comme le sujet cherchent à atteindre, et avec qui il peut entrer en contact sur un mode jubilatoire, jouissif. (Didier-Weill, 1998, p. 34-42). 


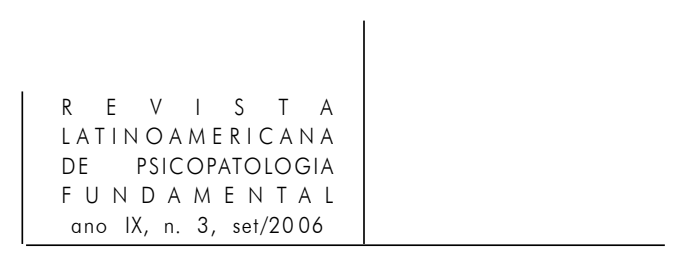

logique de jeu, de création, qui emprunte à l'espace transitionnel, et auquel nous accédons par le biais de la jouissance. Cet excès de plaisir (propre à l'Au-delà du principe du même nom) nous sert de modèle à la fonction thérapeutique du jeu: au travers de la dimension du jouer, persiste celle du jouir, que nous inscrirons du côté de l'éprouvé jubilatoire que procure l'accession à cet espace. Là, le sujet recouvre les dimensions d'Inouï, d'Invisibilité et d'Immatérialité de l'être. Alors et alors seulement, l'interprétation a valeur de castration (et non de frustration) dans la mesure où, prise dans les rets du transfert, elle fait entendre la musique de l'amour. C'est pourquoi la sagesse populaire souligne que si l'on ne connaît pas la chanson, on connaît toujours la musique. Dans cette mesure, elle se constitue en véritable don d'amour - de transfert -: “l'amour c'est donner ce qu'on n’a pas (le signifiant) à quelqu'un qui n’en veut pas” (Lacan, séance du 17 mars 1965), autrement dit à l'analysant, au patient. De fait, pour que le sujet le veuille, l'accepte, il faut un certain espace, "suffisamment bon”, dans lequel se déploie amoureusement le jeu langagier.

Ceci ne va pas sans poser quelques questions sur l'établissement du dispositif, du cadre analytique (setting), de la rencontre clinique.

\section{Pour une clinique transféro-transitionnelle}

Lorsque la prise en charge se définit justement à partir du temps nécessaire à son établissement, c'est la capacité transféro-transitionnelle qui émerge en tant que style de la rencontre, comme de l'intervenant lui-même. Si le signifiant "poulet" émerge à un moment donné du parcours de Mr A., c'est parce que le clinicien obéit à ce modèle temporo-spatial, qui l'autorise psychiquement à poser un acte de parole porteur et révélateur d'une telle capacité: celle qui consiste à faire circuler les signes de pensée langagière. Simultanément à son histoire personnelle, à son parcours professionnel, à ses modèles théorico-cliniques, "ça" vient dans la mesure où le cadre de la rencontre singulière avec $\mathrm{Mr} \mathrm{A}$. permet de se mettre à l'écoute d'autre chose que de son symptôme. C'est donc le style même du sujet, en l'occurrence en place de tenant-lieu de l'Autre, de clinicien, qu'il s'agit ici d'éclairer, à partir de cette intrication réciproque du transfert et de l'espace transitionnel.

"Poulet”, au-delà de l'aspect signifiant caractéristique du transport transférentiel, est aussi le symbole, à l'intérieur de la forme syntaxique empruntée, de l'établissement d'un espace de jeu (langagier), de cette sphère d'expérience au sein de laquelle "I'on peut parler des choses de façon bizarre...”. Il prend effet de réalité et reste susceptible d'appropriation par le sujet, dans la mesure où il 
renvoie à la façon dont l'Autre (maternel) présente le monde à l'enfant (object presenting). Mr A. reconquiert une prise sur le monde qui lui a fait défaut au moment du trauma, qui peut s'apparenter au recouvrement effectif du "sentiment d'omnipotence" de l'enfant, autrement dit de la valence narcissique originelle. Il retrouve également, par le biais de l'interprétation sous transfert, la possibilité créative d'un "déjà là", dans toute sa dimension paradoxale: il ne peut trouver cet espace, que si celui-ci est “réellement” présent, tout comme ce “déjà-là” n’est trouvé que s’il est créé. A ce propos, la question que nous pourrions nous poser est: qui de la poule (et non plus du poulet) ou de l'œuf? L'espace intermédiaire de rencontre induit-il l'émergence du lien la relation transférentielle? Ou bien estce l'établissement d'un lien transférentiel qui contient les germes d'un déploiement transitionnel? En empruntant à Winnicott nous ne pourrons, ni ne voudrons, solutionner ce paradoxe, afin d'en conserver sa valeur praxique. ${ }^{38}$

Dans le cas contraire, comme par exemple lors des prises en charge réalisées par les services d'urgence médico-psychologique à proximité (temporelle et spatiale) de la catastrophe, de l'attentat, un tel modèle ne peut être investi. La rencontre ne peut alors se déplier tant d'un point de vue transitionnel, que transférentiel. La pensée ne circule plus: l'écoute se centre sur la souffrance symptomatique (et non celle du sujet), sur l'évènement traumatique. Il s'agit là d'une clinique hors jeu du transfert...donc hors-jeu tout court. Elle exclut alors également toute potentialité de "régression à la dépendance" au sens où la définit Winnicott (1954, p. 257). ${ }^{39}$ Celle-ci, s'entend comme cette proposition faite au patient par le setting analytique d'expérimenter un climat de holding favorable à un revécu du trauma - non encore intégré car ayant eu lieu précocement. Le point

38. La question se pose de manière identique pour les trois registres lacaniens du réel, de l'imaginaire et du symbolique: lequel des trois est premier? Le réel en tant que bios du corps fœtal? L'imaginaire dans la mesure où le sujet existe dès avant sa naissance, dans le fantasme des parents? Le symbolique dans la mesure où le langage pré-existe et prédétermine ce dernier dans la place dans le monde et dans la généalogie? Impossible de répondre, si ce n’est au travers du constat que faisait Lacan, à savoir l'impossible de parler de l'un des trois registres, sans faire référence au deux autres.

39. “la régression d'un patient est un retour organisé à une dépendance primitive ou une double dépendance. Le patient et le cadre se fondent dans la situation originelle heureuse du narcissisme primaire” (Winnicott, 1954, p. 260).

“Le divan et les coussins sont là pour que la patient s’en serve. Ils apparaîtront dans les idées et les rêves et représenteront alors le corps de l'analyste, ses seins, ses bras, ses mains etc. , de toutes sortes de façons.. Dans la mesure où le patient a régressé (...) le divan, c’est l'analyste" (Winnicott, 1954, p. 261). 


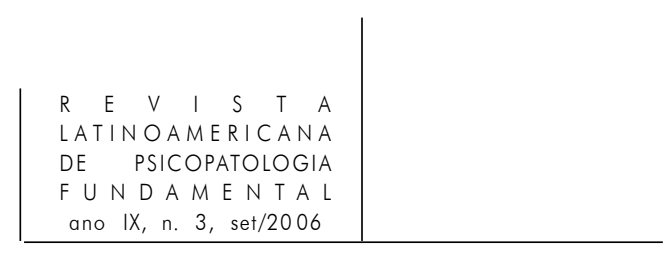

d’importance du cadre de rencontre proposé réside dans la relation de dépendance établi entre l'analysant et l'analysé. Elle inclut en effet une régression langagière, celle-là même que Lacan avait révélé dans l'émergence de signifiants de l'enfance, réactualisés dans le cadre de cette "situation d'interlocution privilégiée" (Gori, 1996, p. 98) du dispositif analytique. Le poulet que l'on passe sur le feu, après l'avoir plumé, afin de le défaire des racines restantes, obéit à cette dimension de l'infantile, scène "potentiellement" (a)perçue alors que nous étions enfant et que nos mères ou leurs substituts, réalisaient dans leurs cuisines. Mr A., alors, n’est plus à poil face au trauma. Il retrouve de sa parure symbolique, après qu'à l'embrasement du sujet par le réel du trauma accidentel, se substitue la flamme du foyer transféro-transitionnel et que de la musique de son crépitement, le je accède à son jeu.

\section{References}

Aristote. Physique. Paris: Les Belles Lettres, 1973. 2 tomes.

Assoun, Paul Laurent. Le mot d'esprit. In: Psychanalyse. Paris: P.U.F., 1997. p. 168-183. (Premier cycle).

Le trauma à l'épreuve de la métapsychologie. Psychiatrie Française “Traumatismes et Sociétés”, v. XXX , n. Sp 99, p. 7-23, 1999.

BoKanowski, Thierry. La conception ferenczienne du traumatisme et ses apports concernant le traumatisme narcissique. Psychiatrie française - "Traumatismes et Sociétés”, v. XXX, n. Sp. 99, p. 71-79, 1999.

CABassut, Jacques. La névrose traumatique ou le nécessaire re-voilement du réel. Psychologie Clinique - "Recherches cliniques en psychanalyse”, Paris: l’Harmattan, Nouvelle série n. 13/ Printemps, p. 191-208, 2002.

Clément, Catherine. Les allumettes et la musique. A propos de l'objet transitionnel. l'Arc, Numéro sur Donald Woods Winnicott. Paris: Duponchelle, 1990. p. 65-69.

Didier-Weill, Alain. Les trois temps de la loi. Paris: Seuil, 1995.

Invocations, Dionysos, Moïse, saint-Paul et Freud. Paris: Calmann-Levy, 1998.

Freud, Sigmund (1905). Le mot d'esprit et sa relation à l'inconscient. Paris: Gallimard, 1988. (Folio-Essais).

(1911). Remarques psychanalytiques sur l'autobiographie d'un cas de paranoïa - Le président Schreber. In: Cinq psychanalyses. Tr. Fr. M. Bonaparte et Loewenstein. Paris: P.U.F., 1954. p. 263- 324. 
(1914). Pour introduire le narcissisme. In: La vie sexuelle. Paris: P.U.F, 1969. p. 81-105. (Bibliothèque de psychanalyse).

(1919). Au delà du principe de plaisir. In: Essais de psychanalyse. Paris: P.B.P, 1980.

(1919). Introduction à la psychanalyse des névroses de guerre. In: Résultats, idées, problèmes I (1890-1920). Paris: P.U.F. p. 243-247. (Petite Bibliothèque de Psychanalyse).

(1926). Inhibition, symptômes et angoisse. Paris: P.U.F., 1981.

(1939). L’homme Moïse et la religion monothéiste. Paris: Gallimard, 1986. (La connaissance de l'inconscient).

Gori, Roland. La preuve par la parole. Paris: P.U.F., 1996. (Psychopathologies).

L’esprit de la langue. Cliniques Méditerranéennes - "Exil et Migrations dans la langue”, Ramonville Saint Agne: Érès, n. 55/56, p. 7-19, 1998.

Green, André. La royauté appartient à l’enfant. L'Arc, Numéro sur Donald W. Winnicott. Paris: Duponchelle, p. 4-12, 1990.

Haм, Mohammed. L'immigré et l'autochtone face à leur exil, cultures d'exclusion et savoirs hors sujet - Essai clinique. Grenoble: P.U.G., 2003.

Julien, Philippe. Psychose, Perversion, Névrose - La lecture de Jacques Lacan. Ramonville Saint Agne: Point hors ligne, Érès, 2000.

LACAN, Jacques (1953). Fonction et champ de la parole et du langage en psychanalyse. In: Ecrits. Paris: Seuil, 1966. p. 237-322.

(1954-55). Le séminaire. Livre II. Le moi dans la théorie de Freud et dans la technique psychanalytique. Paris: Le Seuil, 1978. (Le champ freudien).

(1955-56). Le séminaire. Livre III. Les structures freudiennes dans les psychoses. Paris: Le Seuil, 1981. (Le champ freudien).

(1958). La direction de la cure et les principes de son pouvoir. In: Ecrits. Paris: Seuil, 1966. p. 585-645.

(1963-64). Le séminaire. Livre XI. Les quatre concepts fondamentaux de la psychanalyse. Paris: Seuil, 1973. Inédit.

(1964-65). Le séminaire. Livre XII. Problèmes cruciaux pour la psychanalyse.

(1968-69). Le séminaire. Livre XVI. D’un Autre à l'autre. Inédit.

La troisième. Lettres de l'Ecole Freudienne, Bulletin intérieur de l'Ecole Freudienne de Paris, VII ème Congrès de l'Ecole Freudienne, Rome, n. 16, p. 177-203, Nov./1975. 


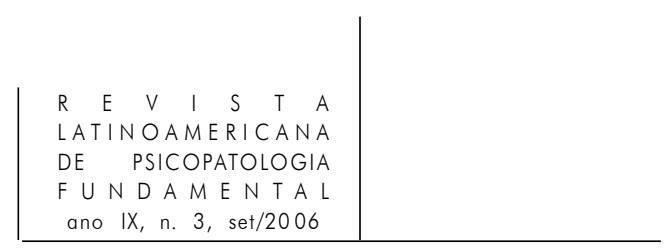

(1975-76). Le séminaire. Livre XXIII. Le sinthome. Inédit.

Laplanche, Jean. Nouveaux fondements pour la psychanalyse. Paris: P.U.F., 1987.

Lekeuche, Philippe. Le test de Szondi comme outil psychothérapeutique dans des cas de toxicomanies. Psychiatrie et existence, Décade de Cérisy. Textes réunis par P. Fédida et J. Schotte. Jérôme Millon Ed., Collection Kriss, p. 321-337, Sept./1989.

Mannoni, Maud. La théorie comme fiction. Paris: Seuil, Points-Essais, 1989.

Mannoni, Octave. La part du jeu. L'Arc, Numéro sur Donald W. Winnicott. Paris: Duponchelle, p. 39-45, 1990.

Tовоul, Bernard. Remarques introductives à la question du trauma, Figures de la psychanalyse. Logos-Anankè - Trauma, Erès, Ramonville Saint-Agne, n. 8, p. 9-13, 2003.

VIVEs, Jean-Michel. Leurre et trompe-l'œil dans l'art et la psychanalyse. Essaim, Toulouse: Érès, n. 4, p. 27-44, 1999.

WinTER, Jean-Pierre. Le traumatisme entre réel et réalité. Les carnets de psychanalyse, n. 5, p. 212-20, 1994.

WinnicotT, Donald Woods (1954). Les aspects métapsychologiques et cliniques de la régression au sein de la situation analytique. In: De la pédiatrie à la psychanalyse. Paris: Sciences de l'homme Payot, 1969. p. 250-267.

(1967). Le concept d’individu sain. In: Conversations ordinaires. Paris: N.R.F./ Gallimard, 1988. p. 23-42. (Connaissance de l’inconscient, dirigée par J.-B. Pontalis).

(1971). Jeu et réalité. L'espace potentiel. Paris: N.R.F/Gallimard, 1975. (Connaissance de l’inconscient, dirigée par J.-B. Pontalis).

(1986). Conversations ordinaires. Paris: N.R.F./Gallimard, 1988. (Connaissance de l’inconscient, dirigée par J.-B. Pontalis).

\section{Resumos}

A abordagem freudiano-lacaniana, paralelamente ao mau encontro do real, define o trauma como efeito da linguagem potencialmente estruturante para o sujeito. $O$ pensamento winnicottiano, paradoxal por excelência, dedica ao brincar e à ligação precoce do infans com a "boa mãe" um lugar central em detrimento da pulsão, da sexualidade infantil, do aspecto linguageiro ou do papel do pai com relação ao trauma. Formulando um espaço transferencial-transicional de encontro, os autores propõem combinar diferentes aspectos do heurístico e da práxis psicanalítica, sustentados por estas duas correntes. Bebendo em um e outro destes aportes, mostrarão sua pertinência 
de articulação com relação à clínica do trauma. Um fragmento de caso do Sr. A, neurótico traumático, servirá de base para este propósito.

Palavras-chave: Brincar, objecto a/objeto transicional, transferência, trauma, chiste

El abordaje freudiano-lacaniano, paralelamente al mal encuentro con lo real, define el trauma como efecto de lenguaje potencialmente estructurante del sujeto. El pensamiento winnicottiano, paradojal por excelencia, dedica al juego y a la ligadura precoz del infans con la "buena madre" un lugar central, en detrimento de la pulsión, la sexualidad infantil, del aspecto lenguajero o del papel del padre en lo concerniente al trauma. Formulando un espacio transferencial-transicional de encuentro, los autores proponen combinar diferentes aspectos de lo heurístico y de la praxis psicoanalítica, sustentados por estas dos tendencias. Bebiendo en uno y en otro de esos aportes, mostraran su pertinencia de articulación con relación a la clínica del trauma. Un fragmento del caso del sr. A, neurótico traumático, servirá de base para este propósito. Palabras clave: Jugar, objeto a/objeto transicional, transferencia, trauma, chiste

In conjunction with the encounter with the real, the Freudian-Lacanian approach defines trauma as the effect of potentially structuring language for the subject. Winnicott's thought, paradoxical par excellence, gives a central place to playing and to the early bonding of the infans with its "good mother," considering them more important than the drive, infantile sexuality, aspects related to language, or the role of the father in relation to the trauma. By formulating a transferential-transitional space for encounter, the authors of this article combine different aspects of heuristics and psychoanalytic praxis, sustained by these two currents. Based on these sources, the authors show the pertinence of articulating these sources in the clinic of the trauma. A fragment of the case of Mr. A, a traumatic neurotic, serves as the basis for this proposal.

Key words: Play, object $a /$ transitional object, transference, trauma, Witz 